\title{
Decrease in the matrix enhancement effect on pesticides analysis with GC-MS using new types of solid-phase extraction column
}

\author{
Kuniyo Sugitate ${ }^{1, *}$ and Machiko SaKa ${ }^{2}$ \\ ${ }^{1}$ Agilent Technologies Japan, Ltd., 9-1 Takakura-machi, Hachioji-shi, Tokyo 192-8510, Japan \\ ${ }^{2}$ The Institute of Environmental Toxicology, 1-12-11 Hanakoganeiminami-cho, Kodaira-shi, Tokyo 187-0003, Japan
}

(Received February 5, 2015; Accepted March 19, 2015)

\begin{abstract}
Matrix removal ability of several new types of Solid Phase Extraction (SPEs) column was tested. E-HyCu is made of new type of material, chemically-modified carbon fibers, and can remove food components, monoacylglycerols, tocopherols, and sterols, etc. Z-Sep+ and Z-Sep/C18 are also new types of materials that contain zirconium dioxide. Z-Sep+ and Z-Sep/C18 are used for high lipid samples as the dispersive SPE in the QuEChERS method. We evaluated these sorbents as filled up columns. In our previous study, we reported that when the analytical method based on the multiresidue method is applied, monoacylglycerols are the most significant components that cause a matrix enhancement effect on pesticides in food using GC-MS. These new types of SPEs could remove not only monoacylglycerols, but also fatty acids, tocopherols, flavonoids, and sterols. The matrix enhancement effects of approximately 260 pesticides spiked in brown rice extracts pretreated with these SPEs were dramatically reduced. (C) Pesticide Science Society of Japan

Keywords: matrix effect, GC-MS, pesticide analysis, E-HyCu, Z-Sep+, Z-Sep/C18.
\end{abstract}

Electronic supplementary materials: The online version of this article contains supplementary materials (Supplemental Tables S1 and S2), which are available at http://www.jstage.jst.go.jp/browse/jpestics/

\section{Introduction}

The matrix enhancement effects has been a critical problems in pesticide residue analysis for the quantification of trace ppb levels. ${ }^{1-5)}$ In our previous study, ${ }^{6}$ ) the matrix components of different types of agricultural products, which were pretreated using the Multiresidue Method for Agricultural Chemicals by GC-MS (Agricultural Products) in the "Analytical Method for Residual Compositional Substances of Agricultural Chemicals, Feed Additives, and Veterinary Drugs in Food" under the Japanese Positive List System (JPL) of Ministry of Health, Labour and Welfare (hereafter, called the JPL method), were examined and some common matrices, monoacylglycerols, tocopherols, and sterols, were found in the sample solution. We also concluded that the monoacylglycerols were the most significant component among them. The matrix effect of the brown rice extract was highest among 5 different agricultural products, and the concentration of monoacylglycerols was also highest.

Recently, some new types of Solid Phase Extraction (SPEs) have been developed. One was the E-HyCu, which was developed by Ehime University, ${ }^{7)}$ and others were Z-Sep+ and Z-Sep/

\footnotetext{
* To whom correspondence should be addressed.

E-mail: kuniyo_sugitate@agilent.com

Published online June 4, 2015

(c) Pesticide Science Society of Japan
}

C18 by Sigma-Aldrich. ${ }^{8)}$ E-HyCu is made of carbon fibers with a chemical modification, but the details of this column have not been clarified due to a pending patent application. This column has a feature that converts the current 2-step column purification into one step, in which E-HyCu plays the role of both $\mathrm{C} 18$ and Carbon/ $\mathrm{NH}_{2}$. On the other hand, the Z-Sep series has a stationary phase of silica gel and zirconium dioxide bound to its surface. Mono/di/tri glycerols are combined with these SPEs by a Lewis acid-base interaction. ${ }^{8)} \mathrm{Z}-\mathrm{Sep}+$ is a column in which both Z-Sep and C18 are chemically bonded to silica gel, and Z-Sep/C18 is a column in which both Z-Sep and C18 are mixed. Lozano et al. ${ }^{9)}$ and Rajski et al. ${ }^{10)}$ reported that they applied these SPEs to samples with high oil content, i.e., avocados and almonds, using the QuEChERS method. Their approach was to use the QuEChERS method with ethyl acetate extraction and Z-Sep for cleanup. The tested pesticides included 166 compounds for GC-MS/MS and 116 compounds for LC-MS/ MS. They concluded that the Z-Sep series removed more matrix components than did the PSA and C18.

The purpose of this study was to evaluate whether these new types of SPEs are capable of removing monoacylglycerols, which are the most significant matrices of the matrix effect, and whether the matrix effect is reduced if monoacylglycerols are removed. We tested these SPEs in combination with the JPL method and evaluated the removal ability of matrices and the matrix enhancement effect. The elution rates of approximately 260 pesti- 
cides from these SPEs were also tested.

\section{Materials and Methods}

1. Experiment 1: Matrix removal ability using a mixed standard solution for evaluation

1-Monopalmitin, palmitic acid, oleic acid, linoleic acid, and stigmasterol were obtained from Tokyo Chemical Industry Co., Ltd. (Tokyo, Japan). 1-Monomyristin, 1-monostearin, $\alpha$-tocopherol, quercetin, and phytol were obtained from Wako Pure Chemical Industries, Ltd. (Osaka, Japan). 1-Monopalmmitin, 1-monomyristin, and 1-monostearin were the representative components for the monoacylglycerols; palmitic acid, oleic acid, and linoleic acid were those of the fatty acids; stigmasterol was that of the sterols; $\alpha$-tocopherol was that of the tocopherols; and quercetin was that of the flavonoids. Phytol was also used because it remained in the sample solution from green and yellow vegetables, such as spinach. $\left.{ }^{6}\right)$ These chemicals were mixed and used as matricesmixed standard solution. Each concentration in the solution was from 100 to $1000 \mathrm{mg} / \mathrm{L}$ and was diluted for the SPE treatment. The loading amounts of each monoacylglycerol and quercetin were $40 \mathrm{ng}$, and those of phytol, stigmasterol, alpha-tocopherol and each fatty acid were $80 \mathrm{ng}, 200 \mathrm{ng}, 400 \mathrm{ng}, 400 \mathrm{ng}$, respectively. These concentrations were estimated based on our previous study. ${ }^{6)}$ Although fatty acids are almost completely removed using $\mathrm{NH}_{2}$ or PSA in the JPL method, they were added to the matricesmixed standard solution because fatty acids were the compound most present in the sample solution before SPE cleanup.

Z-Sep+ (500 mg per pack) and Z-Sep/C18 (120 mg/300 mg per pack) were obtained from Sigma-Aldrich Japan (Tokyo, Japan). One cartridge was filled with two packs of Z-Sep+ or $\mathrm{Z}$-Sep/C18. These SPEs were used as substitutes for C18 in the JPL method.

E-HyCu was obtained from SEED TEC (Ehime, Japan, it is now commercialized by the Morinaga Institute of Biological Science, Inc. (Kanagawa, Japan)). Bond Elut C18 (1 g), Carbon/ $\mathrm{NH}_{2}(500 \mathrm{mg} / 500 \mathrm{mg})$, and Carbon/PSA $(500 \mathrm{mg} / 500 \mathrm{mg})$ were obtained from Agilent Technologies Japan (Tokyo, Japan).

The extraction solvent was a mixture of toluene and acetonitrile $(50 \mathrm{~mL})$ for $\mathrm{E}-\mathrm{HyCu}$, and the blend ratio was based on a report by Ehime University. ${ }^{7)} \mathrm{Z}$-Sep+ and Z-Sep/C18 were used as substitutes for $\mathrm{C} 18$ as was described earlier, so acetonitrile $(20 \mathrm{~mL})$ was used for the extraction solvent. For Carbon/ $\mathrm{NH}_{2}$ and Carbon/PSA, toluene: acetonitrile (Tol/ACN) $(1: 3, \mathrm{v} / \mathrm{v}$, $20 \mathrm{~mL}$ ) was used according to the JPL method.

The flowchart of the JPL method using these SPE is shown in Fig. 1.

The eluted matrices-mixed standard solutions from each SPE were dried and derivatized using $N$-methyl- $N$-trimethylsilyltrifluoroacetamide (MSTFA) with 1\% trimethylchrolosilane (TMCS) in pyridine hydrate. ${ }^{11)}$ An Agilent 7890B gas chromatograph equipped with a 5977 mass spectrometer and a 7693A autoinjector was used to find the removal rate of these matrices. The operating conditions were as follows: injection liner-split, single taper, ultra inert for low pressure with glass wool (Agilent

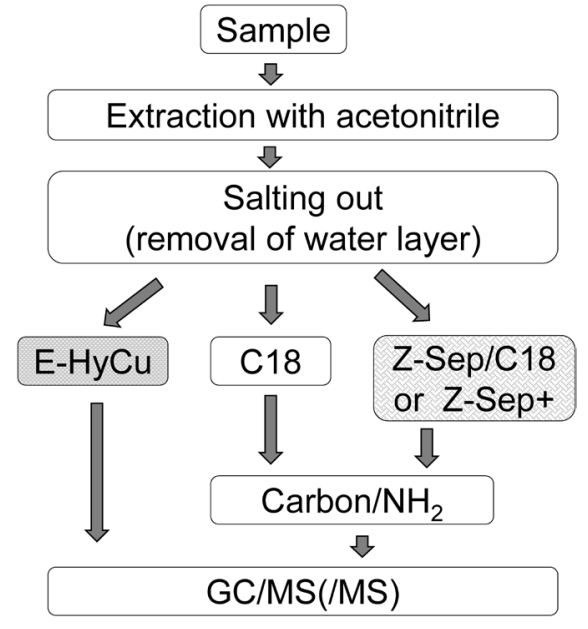

Fig. 1. Flow chart of the sample preparation using three different types of SPE.

Technologies, p/n 5190-2295); column-Duragurd column (nonpolar deactivated pre-column connected directly to DB-5ms, $10 \mathrm{~m}$ ), DB- $5 \mathrm{~ms}, 30 \mathrm{~m} \times 0.25 \mathrm{~mm}$ i.d., $0.25 \mu \mathrm{m}$ thickness, (Agilent Technologies); oven temperature program $-60^{\circ} \mathrm{C}$ ( 1 min hold) to $325^{\circ} \mathrm{C}$ at $10^{\circ} \mathrm{C} / \mathrm{min}\left(10 \mathrm{~min}\right.$ hold); injector temperature $-250^{\circ} \mathrm{C}$; injection mode-split (split ratio, 10:1); carrier gas-Helium $1.1 \mathrm{~mL} / \mathrm{min}$ constant flow; transferline $-290^{\circ} \mathrm{C}$; ion source temperature- $250^{\circ} \mathrm{C}$; mass mode-scan $(\mathrm{m} / z 50-650)$.

\section{Experiment 2: Matrix removal efficiency using brown rice} Brown rice (Hitomebore) was tested as a real sample because it showed the highest matrix effect. ${ }^{6}$ The sample preparation followed the JPL method.

A $10 \mathrm{~g}$ brown rice sample was homogenized with $50 \mathrm{~mL}$ of acetonitrile after soaking in $20 \mathrm{~mL}$ of water for $15 \mathrm{~min}$. The sample was suction filtered. The residue was extracted again with $20 \mathrm{~mL}$ of acetonitrile, and then suction filtered again. Both filtrates were mixed. Acetonitrile was added to make a $100 \mathrm{~mL}$ solution, and $20 \mathrm{~mL}$ (equivalent to a $2 \mathrm{~g}$ sample) was measured. To the sample solution was added $10 \mathrm{~g}$ of sodium chloride and $20 \mathrm{~mL}$ of $0.5 \mathrm{~mol} / \mathrm{L}$ phosphate buffer $(\mathrm{pH} 7.0)$. The mixed solution was shaken for $10 \mathrm{~min}$, and the aqueous layer was discarded. The acetonitrile layer was used for each SPE. After the SPE cleanup, all eluates were evaporated to dryness at $40^{\circ} \mathrm{C}$, and then dissolved in acetone $/ n$-hexane $(1: 1, \mathrm{v} / \mathrm{v})$ to make a $1 \mathrm{~mL}$ solution. The derivatization procedure and analysis conditions were the same as for Experiment 1.

\section{Experiment 3: Evaluation of the matrix enhancement effect in} brown rice

The pesticide mixed standard solutions, including Pesticides standard solution 31 (85 pesticides), 48 (61 pesticides), 51 (26 pesticides), 61 (49 pesticides), and 63 (44 pesticides) were obtained Kanto Chemical Co., Inc. (Tokyo, Japan). Approximately 260 pesticides were spiked at $100 \mathrm{ng} / \mathrm{mL}$ into brown rice extract. To avoid the influence of dryness, pesticides were spiked into the 
brown rice extract with a syringe. The standard solution for evaluating the matrix effect was made in the same way, that is, pesticides were spiked into the organic solvent with a syringe. An Agilent 7890B gas chromatograph equipped with a 7000C triple quadrupole mass spectrometer and a 7693A autoinjector was used to evaluate the multiple pesticides. The analysis conditions were as follows: injection linear-splitless, Siltek deactivated liner (borosilicate glass) (Restek, Bellefonte, PA, USA) (discontinued); column-factorFOUR VF- $5 \mathrm{~ms}, 30 \mathrm{~m} \times 0.25 \mathrm{~mm}$ i.d., $0.25 \mu \mathrm{m}$ thickness (Agilent Technologies); oven temperature program$70^{\circ} \mathrm{C}(2 \mathrm{~min}$ hold $)$ to $150^{\circ} \mathrm{C}$ at $25^{\circ} \mathrm{C} / \mathrm{min}$, to $200^{\circ} \mathrm{C}$ at $3^{\circ} \mathrm{C} / \mathrm{min}$, then, to $310^{\circ} \mathrm{C}$ at $8^{\circ} \mathrm{C} / \mathrm{min}(5 \mathrm{~min}$ hold); injector temperature$250^{\circ} \mathrm{C}$; injection mode-pulsed splitless (25 psi, $1 \mathrm{~min}$ ); carrier gas-Helium $1.1 \mathrm{~mL} / \mathrm{min}$ constant flow; transferline $-280^{\circ} \mathrm{C}$; ion source temperature $-300^{\circ} \mathrm{C}$; mass mode-MRM (Multiple Reaction Monitoring).

The GC-MS/MS measurement order was performed as follows.

\section{Pesticide standard}

$$
\rightarrow \text { sample solution ( } n=3 \text {, continuously) } \rightarrow \text { solvent }
$$

The pesticide mixed standard solution was initially analyzed. After checking the response stability of standard solution, the sample solution, which had been spiked with the pesticide standard solution, was then continuously analyzed three times. Before moving to the next sample solution, the solvent (acetone: $n$ hexane, $1: 1, \mathrm{v} / \mathrm{v}$ ) was injected several times to avoid any influence from the previous sample. After checking the response, the stability, and the peak shape of the next standard, the next sample solution was analyzed. The matrix effect value of the pesticide in the sample solutions was evaluated as the relative response of the pesticides in the sample solution to those in the standard solution.

4. Experiment 4: Elution rate of pesticide using each SPE

The elution rates of approximately 260 pesticides from each SPE were obtained in order to evaluate whether they could be used

Table 1. Matrix removal rate (\%) from $\mathrm{E}-\mathrm{HyCu}$

\begin{tabular}{|c|c|c|c|}
\hline Compounds & $\begin{array}{c}\text { E-HyCu } \\
\left.\text { Tol/ACN }{ }^{a}\right) \\
(5: 95, v / v)\end{array}$ & $\begin{array}{c}\text { E-HyCu } \\
\text { Tol/ACN }{ }^{\mathrm{a})} \\
(10: 90, \mathrm{v} / \mathrm{v})\end{array}$ & $\begin{array}{c}\text { E-HyCu } \\
\text { Tol/ACN }{ }^{\mathrm{a})} \\
(25: 75, \mathrm{v} / \mathrm{v})\end{array}$ \\
\hline Palmitic acid & 99.8 & 99.7 & 99.6 \\
\hline Phytol & 61.1 & 44.2 & 40.9 \\
\hline Linoleic acid & 99.9 & 99.8 & 99.8 \\
\hline Oleic acid & 99.8 & 99.7 & 99.7 \\
\hline 1-Monomyristin & 83.2 & 65.1 & 60.9 \\
\hline 1-Monopalmitin & 97.3 & 81.6 & 72.1 \\
\hline 1-Monostearin & 97.9 & 94.4 & 81.1 \\
\hline$\alpha$-Tocopherol & 88.9 & 64.8 & 49.5 \\
\hline Quercetion & 100 & 100 & 100 \\
\hline Stigmasterol & 100 & 98.3 & 70.9 \\
\hline
\end{tabular}

a) Eluate-toluene/acetonitrile, $50 \mathrm{~mL}$. for the multiresidue analysis. The pesticide mixed standard solution in acetonitrile was used for evaluating the elution rate. The analysis conditions were the same as those for Experiment 3.

\section{Results and Discussion}

1. Experiment 1: Matrix removal ability using a mixed standard solution for evaluation

The matrix removal ability of E-HyCu is shown in Table 1. Almost $100 \%$ of fatty acids and quercetin (flavonoid) were removed, ranging from Tol/ACN (5:95, v/v) to Tol/ACN $(25: 75$, $\mathrm{v} / \mathrm{v})$. Approximately $90-100 \%$ of the other matrices, monoacylglycerols, tocopherol, and sterol, were removed with Tol/ACN $(5: 95, \mathrm{v} / \mathrm{v})$; otherwise, the removal rate decreased as the ratio of toluene increased. The removal ability of phytol was approximately $60 \%$ with Tol/ACN (5:95, v/v) and $40 \%$ with Tol/ACN $(25: 75, \mathrm{v} / \mathrm{v})$.

The removal abilities of Z-Sep+, Z-Sep/C18, and Bond Elut C18 are shown in Table 2. Bond Elut C18 is adopted as an SPE that can remove fats in the JPL method. Although Bond Elut C18 showed an ability to remove $100 \%$ of sterols, the removal rates of the other matrices were less than 50\%. Z-Sep+ and Z-Sep/C18 showed higher removal abilities than did Bond Elut C18.

Table 2. Matrix removal rate (\%) from Z-Sep+, Z-Sep/C18 and C18

\begin{tabular}{lccc}
\hline Compounds & Z-Sep ${ }^{\mathrm{a})}$ & Z-Sep/C18 & C18 \\
\hline Palmitic acid & 99.6 & 100 & 26.1 \\
Phytol & 93.7 & 90.0 & 29.4 \\
Linoleic acid & 98.1 & 100 & 26.8 \\
Oleic acid & 99.0 & 99.9 & 24.6 \\
1-Monomyristin & 99.2 & 100 & 32.1 \\
1-Monopalmitin & 99.2 & 100 & 33.8 \\
1-Monostearin & 93.8 & 99.3 & 46.0 \\
$\alpha$-Tocopherol & 94.3 & 99.5 & 48.2 \\
Quercetion & 98.6 & 100 & 16.0 \\
Stigmasterol & 100 & 100 & 100 \\
\hline
\end{tabular}

a) Eluate-acetonitrile, $20 \mathrm{~mL}$.

Table 3. Matrix removal rate (\%) from Carbon/PSA and Carbon/ $\mathrm{NH}_{2}$

\begin{tabular}{lcc}
\hline \multicolumn{1}{c}{ Compounds } & Carbon/PSA ${ }^{\mathrm{a})}$ & Carbon/ $\left.\mathrm{NH}_{2}{ }^{\mathrm{a}}\right)$ \\
\hline Palmitic acid & 100 & 100 \\
Phytol & 24.8 & 41.4 \\
Linoleic acid & 100 & 100 \\
Oleic acid & 100 & 100 \\
1-Monomyristin & 36.7 & 52.0 \\
1-Monopalmitin & 33.5 & 49.9 \\
1-Monostearin & 45.8 & 58.9 \\
$\alpha$-Tocopherol & 40.7 & 49.6 \\
Quercetion & 100 & 100 \\
Stigmasterol & 99.3 & 99.9 \\
\hline
\end{tabular}

a) Eluate-toluene/acetonitrile (1:3, v/v), $20 \mathrm{~mL}$. 


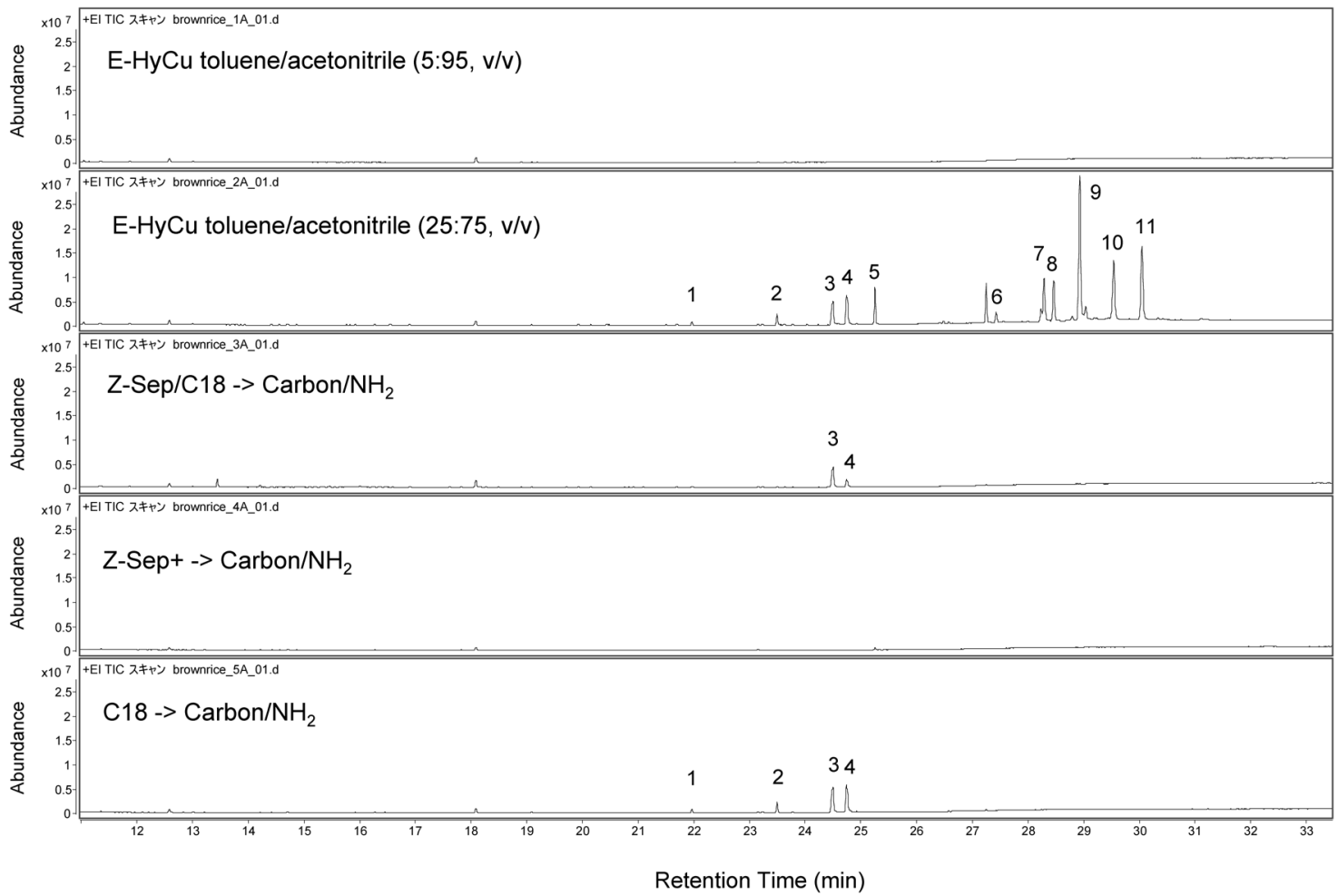

Fig. 2. TICC of brown rice extraction with each sample preparation. 1. 1-monomyristin, 2. 1-monopalmitin, 3. 2-monoolein, 4. 1-monoolein, 5. squalane, 6. $\alpha$-tocopherol, 7. campesterol, 8. stigmasterol, 9. $\beta$-sitosterol, 10. cycloartenol, 11. 24-methylenecycloartan-3 $\beta$-oyl acetate.

We also examined Carbon/ $\mathrm{NH}_{2}$ and Carbon/PSA, which are sometimes used instead of Carbon/ $\mathrm{NH}_{2}$, because these layered SPEs are used after C18 in the JPL method. As a result, both SPEs removed about $100 \%$ of the fatty acids, quercetin (flavonoid), and sterols. However, the removal rate of phytol was not sufficient (Table 3).

\section{Experiment 2: Matrix removal efficiency using brown rice} Three different types of sample treatments-one following the JPL method, the others using the E-HyCu and Z-Sep serieswere applied to brown rice. The total Ion Current Chromatogram (TICC) of each treatment is shown in Fig. 2. Several monoacylglycerols remained in the sample solution after treatment with the JPL method, a result consistent with our previous study ${ }^{6}{ }^{6}$ A small amount of monoacylglycerols was also observed in the sample solution with Z-Sep/C18 and Z-Sep+, both followed by Carbon/ $\mathrm{NH}_{2}$. In this case, in the sample solution that was treated by E-HyCu with Tol/ACN $(25: 75$, v/v), not only the monoacylglycerols but also alpha-tocopherol and several sterols remained. The amount of the residual matrix components was semiquantifited using the mixed standard solution shown in Experiment 2. The total concentration of monoaclyglycerols in the sample solution that was treated using the JPL method was $114 \mathrm{mg} / \mathrm{L}$. The total concentration of monoacylglycerols in the sample solution that was treated using Z-Sep+, Z-Sep/C18 (both followed by Carbon/ $\mathrm{NH}_{2}$ ), E-HyCu with $5 \% \mathrm{~T} / \mathrm{A}$, and $\mathrm{E}-\mathrm{HyCu}$ with Tol/ACN $(25: 75, \mathrm{v} / \mathrm{v})$ were $3.6 \mathrm{mg} / \mathrm{L}, 46 \mathrm{mg} / \mathrm{L}, 0.3 \mathrm{mg} / \mathrm{L}$, and $114 \mathrm{mg} / \mathrm{L}$, respectively. Furthermore, the remaining alphatocopherol and sterols in the sample solution using $\mathrm{E}-\mathrm{HyCu}$ with Tol/ACN $(25: 75, \mathrm{v} / \mathrm{v})$, were $6.5 \mathrm{mg} / \mathrm{L}$ and $484 \mathrm{mg} / \mathrm{L}$, respectively. The result of the matrix removal efficiency is as follows.

E-HyCu with Tol/ACN (5:95, v/v) $>$ Z-Sep + >Z-Sep/ C18 $>$ Bond Elut C18 $>$ E-HyCu with Tol/ACN $(25: 75$, v/v)

3. Experiment 3: Evaluation of the matrix enhancement effect in brown rice

The list of the matrix effect values using different types of SPEs is shown in Supplemental Table 1. Our previous research demonstrated that the matrix effects were high in pesticides that eluted in the second half of a chromatogram. ${ }^{6}$ Based on this result, we investigated the matrix effects of 89 pesticides that were eluted after mepronil, that is, pesticides eluted just after 1-monomyristin; the mean of the matrix effect values of $\mathrm{E}-\mathrm{HyCu}$ (with Tol/ ACN (5:95, v/v)), E-HyCu (with Tol/ACN (25:75, v/v)), Bond Elut C18, Z-Sep+ and Z-Sep/C18 (Bond Elut C18, Z-Sep+ and $\mathrm{Z}$-Sep/C18 were followed by Carbon/ $\mathrm{NH}_{2}$ ) were $112 \%, 218 \%$, $209 \%, 144 \%$ and $151 \%$, respectively. The matrix effect value using E-HyCu with Tol/ACN (5:95, v/v) and the Z-Sep series were dramatically reduced as compared to that using the JPL method (using $\mathrm{C} 18$ followed by Carbon/ $\mathrm{NH}_{2}$ ). Although EHyCu with Tol/ACN $(25: 75$, v/v) eluted not only the monoacylglycerols but also the sterols and other matrices, it showed matrix effect values similar to those of the JPL method. This means that the quantity of monoacylglycerols contributed most to the 


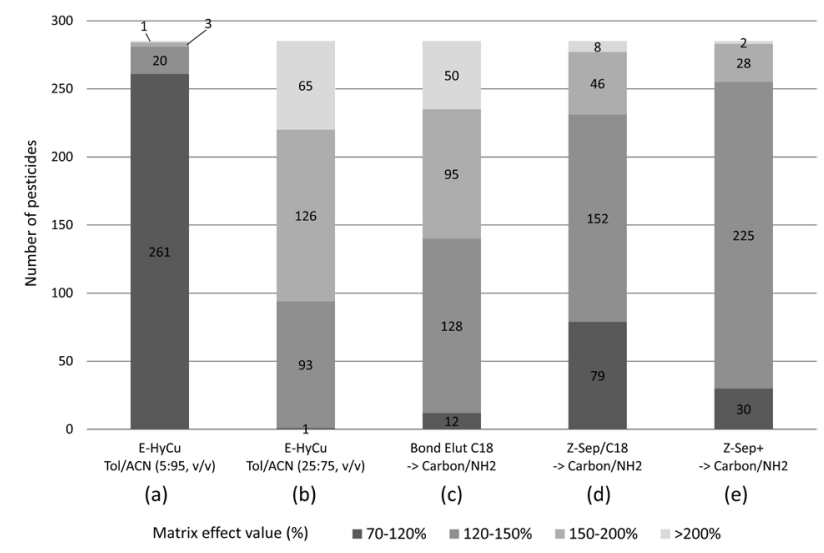

Fig. 3. Distribution chart of the number of the pesticides classified according to matrix effect value. (a) toluene/acetonitrile $(5: 95, \mathrm{v} / \mathrm{v}), 50 \mathrm{~mL}$, (b) toluene/acetonitrile $(25: 75, \mathrm{v} / \mathrm{v}), 50 \mathrm{~mL}$, (c) Bond Elut-acetonitrile, $20 \mathrm{~mL}$, Carbon/ $\mathrm{NH}_{2}$-toluene/acetonitrile (1:3, v/v), $20 \mathrm{~mL}$, (d) Z-Sep/ C18-acetonitrile, $20 \mathrm{~mL}$, Carbon/ $\mathrm{NH}_{2}$-toluene/acetonitrile $(1: 3, \mathrm{v} / \mathrm{v})$, $20 \mathrm{~mL}$, (e) Z-Sep+-acetonitrile, $20 \mathrm{~mL}$, Carbon/ $\mathrm{NH}_{2}$-toluene/acetonitrile $(1: 3, \mathrm{v} / \mathrm{v}), 20 \mathrm{~mL}$

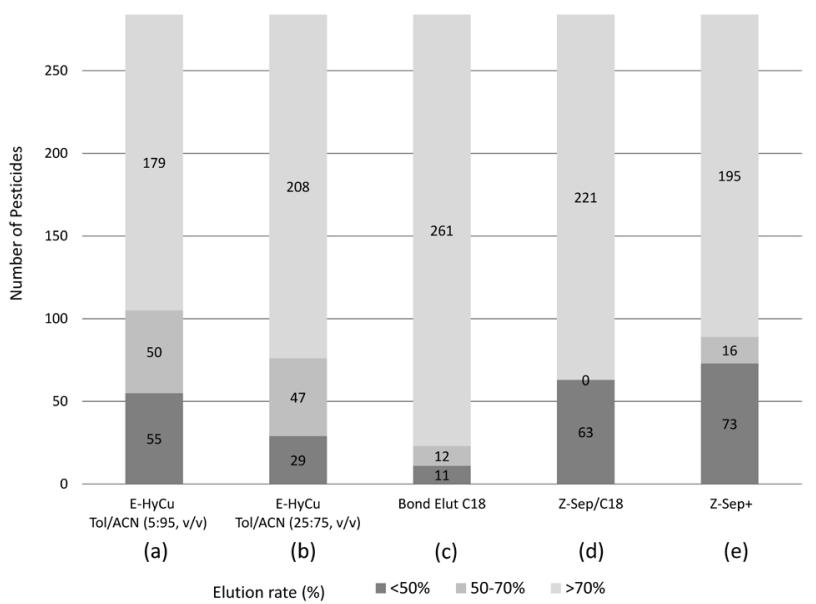

Fig. 4. Distribution chart of the number of pesticides classified according to elution rate from each SPE. (a) toluene/acetonitrile $(5: 95, \mathrm{v} / \mathrm{v})$, $50 \mathrm{~mL}$, (b) toluene/acetonitrile $(25: 75, \mathrm{v} / \mathrm{v}), 50 \mathrm{~mL}$, (c) Bond Elut-acetonitrile, $20 \mathrm{~mL}$, (d) Z-Sep/C18-acetonitrile, $20 \mathrm{~mL}$, (e) Z-Sep+-acetonitrile, $20 \mathrm{~mL}$.

matrix effects, which coincides with our previous study. A distribution graph of approximately 260 pesticides (actually 286 pesticides because some pesticides contain isomers) is shown in Fig. 3. This graph shows that the matrix effect values of more than $98 \%$ of the pesticides were less than $150 \%$ using E-HyCu with Tol/ACN $(5: 95, \mathrm{v} / \mathrm{v})$, while the matrix effect values of around $90 \%$ and $80 \%$ of the pesticides were less than $150 \%$ using Z-Sep+ and Z-Sep/C18, respectively.

\section{Experiment 4: Elution rate of pesticide using each SPE}

Since the relationship between the matrix removal ability and the matrix effect was clarified in Experiment 1-3, the elution rate of pesticides from the different types of SPEs was exam- ined to determine which pesticide can be applied. A distribution graph of elution rate for about 260 pesticides is shown in Fig. 4. Details of data are shown in Supplemental Table 2. The elution rate of pesticides with a planar structure (e.g., quinoclamine, quinomethionate, cinidon-ethyl) and pyrethroids (e.g., cypermethrin, fenvarelate, dertamethrin) were less than $30 \%$ using E-HyCu. Otherwise, the elution rate of organophosphorus compounds, which all contained the $\mathrm{P}=\mathrm{O}$ group (e.g., ethoprophos, dimethylvinphos, fenamiphos) and triazole fungicide (e.g., triadimenol, microbutanil, uniconazole) was almost zero using the Z-Sep series. Z-Sep/C18 showed a higher elution rate than Z-Sep+ in some pesticides, such as cadusafos, iprobenphos, diphenamid, and napropamide. All but 4 pesticides (tricyclazole, cyproconazole, pyraclofos and imibenconazole) had a good elution rate with any column.

The matrix removal efficiency was examined by $\mathrm{E}-\mathrm{HyCu}$, Z-Sep+, and Z-Sep/C18 using the JPL method. The E-HyCu with Tol/ACN $(5: 95, \mathrm{v} / \mathrm{v})$ eluate showed the best removal efficiency. E-HyCu with Tol/ACN (5:95, v/v), Z-Sep+, and Z-Sep/ C18 have the ability to remove not only the monoacylglycerols, which were the component causing the matrix effect, but also fatty acids, flavonoids, tocopherols, and sterols. Using these new SPEs dramatically reduced the matrix effect. However, it is necessary to select the proper SPE because some characteristic pesticides show a low elution rate. Furthermore, when we used Z-Sep+ and Z-Sep/C18 as a substitute for C18 in the JPL method, there seems to be a different way to use them. For example, when Z-Sep+ or Z-Sep/C18 is connected to the multi-layer column (Carbon/ $\mathrm{NH}_{2}$ ) in the JPL method, the eluate should be Tol/ACN $(1: 3, \mathrm{v} / \mathrm{v})$. Therefore, further improvement in the analytical method and inspection of other agricultural products is needed.

\section{References}

1) J. Hajšlová and J. Zrostlíková: J. Chromatogr. A 1000, 181-197 (2003).

2) M. Anastassiades, K. Maštovská and S. J. Lehotay: J. Chromatogr. A 1015, 163-184 (2003).

3) T. Čajka, K. Maštovská, S. J. Lehotay and J. Haǰ̌lová: J. Sep. Sci. 28, 1048-1060 (2005).

4) C. Sánchez-Brunete, B. Albero, G. Martin and J. L. Tadeo: Anal. Sci. 21, 1291-1296 (2005).

5) K. Maštovská, S. J. Lehotay and M. Anastassiades: Anal. Chem. 77, 8129-8137 (2005).

6) K. Sugitate, S. Nakamura, N. Orikata, K. Mizukoshi, M. Nakamura, A. Toriba and K. Hayakawa: J. Pestic. Sci. 37, 156-163 (2012).

7) Y. Ueda and K. Honda: Abstr. 36th Annu. Meeting Pestic. Resid. Anal. pp. 165-172, 2013 (in Japanese).

8) http://www.sigmaaldrich.com/japan/analytical-chromatography/ sample-preparation/z-sep.html (Accessed 01 Dec., 2014)

9) A. Lozano, Ł. Rajski, S. Uclés, N. Belmonte-Valles, M. Mezcua and A. R. Fernández-Alba: Talanta 118, 68-83 (2014).

10) Ł. Rajski, A. Lozano, A. Uclés, C. Ferrer and A. R. Fernández-Alba: J. Chromatogr. A 1304, 109-120 (2013).

11) Agilent G1676AA Fiehn GC/MS Metabolomics RTL Library, User Guide, Agilent Technologies (2013) 